VoL. 47 (1993) [175-176]

\title{
Compact quantum systems
}

\section{Robert J.B. FawcetT}

This thesis is primarily concerned with compact quantum systems, their relationship to non-compact quantum systems and the classical limit of these systems. A compact quantum system is a quantum mechanical system whose underlying kinematical Lie algebra is a compact algebra instead of the usual non-compact Weyl-Heisenberg algebra $w_{n}$ (of the canonical hermitian position and momentum operators) which can be recovered by a contraction of the compact algebra. It is shown that compact quantum systems can be described in terms of the special orthogonal, unitary and compact sympletic algebras and their representations.

Boson realisations of the compact Lie algebras $s o(n+2), u(n+1)$ and $s p(2 n+2)$ are given, modified slightly from realisations given by Exner, Havlicek and Lassner (Czechoslovak J. Phys. B 26, 1213, 1976) in terms of canonical coordinate and momentun operators. These boson realisations provide a natural generalisation of the Dyson boson realisation of $s o(3)$. They also have a recurrent structure which in the cases of $s o(n+2)$ and $u(n+1)$ allows the explicit construction of any desired irreducible representation entirely in terms of bosons.

Using these boson realisations, a general formulation for compact quantum systems with $n$ degrees of freedom based on $s o(n+2)$ and $u(n+1)$ is given in terms of $n$ compact coordinate and momentum operators $(2 n+1$ degrees of freedom and $2 n+1$ coordinate and position operators in the case of $s p(2 n+2)$ ). The dynamics of such a system is assumed to be determined in the usual way by a Hamiltonian operator, taken here to be a polynomial in the coordinate and momentum operators. Such compact quantum systems can be contracted to corresponding non-compact quantum systems by means of a contraction of the underlying compact kinematical Lie algebra. A new definition of the contraction of a Lie algebra and its representations by the method of sequences of representations is given, suitable to the purposes of this application. The consequences of the definition are explored at length, in particular, with regard to the reducibility and decomposability of the resultant representations of the contracted

Received 19th November, 1990

Thesis submitted to The University of Queensland 7th February 1980. Degree approved 13th November 1980. Supervisor: Professor A.J. Bracken.

Copyright Clearance Centre, Inc. Serial-fee code: 0004-9729/93 \$A2.00+0.00. 
algebra. The principal examples considered are the contractions of $s o(n+2), u(n+1)$ and $s p(2 n+2)$ to $w_{n} \oplus s o(n), w_{n} \oplus u(n)$ and $w_{2 n+1} \oplus s p(2 n)$ respectively, for sequences of both boson and matrix representations. Some simple but hopefully typical examples of compact quantum systems have been investigated in detail, with regard to the behaviour of energy eigenvalues and eigenvectors, and the associated distribution of probability over position, in the contraction limit.

The classical limit of a non-compact quantum system is discussed in connection with the contraction of the kinematical Lie algebra of the system to the abelian algebra of the same dimension. This investigation is extended to compact quantum systems; in particular, the systems based on $s o(3)$ and so(4). With the classical trajectories being recovered in the classical limit, compact classical systems which are the classical limit of corresponding compact quantum systems can be described. The dynamical equations for compact classical systems based on $s o(3), s o(4)$ and $s o(3) \oplus s o(3)$ are given, and the examples of the compact free particle and the compact oscillator are presented in more detail. The so(3) oscillator is solved exactly in terms of Jacobi elliptic functions and it is shown that an so(4) anisotropic oscillator exhibits classical chaos. The relationship of these compact classical systems to ordinary non-compact classical systems is also considered in the context of the relationship of compact quantum systems to noncompact quantum systems.

A conjecture by Peres (Phys. Rev. A 30, 504, 1984) concerning the qualitative properties of matrix elements of representatives of simple quantum observables, in the energy basis of a quantum system whose classical analogue exhibits chaotic behaviour, is tested in the case of a compact quantum system and compared with the results of Feingold, Moiseyev and Peres (Phys. Rev. A 30, 509, 1984) for a quantum spin system. As a result, the validity of the conjecture is questioned.

School of Mathematics

Queensland University of Technology

Brisbane Qld 4000

Australis 